\title{
Transscleral cyclophotocoagulation followed by cataract surgery: a novel protocol to treat refractory acute primary angle closure
}

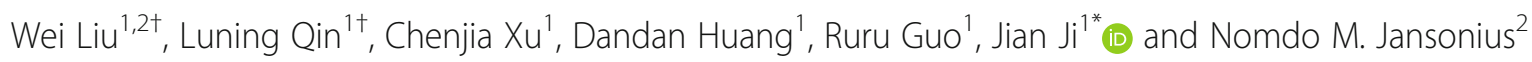

\begin{abstract}
Background: To introduce a novel protocol to treat refractory acute primary angle closure (APAC): transscleral cyclophotocoagulation (TCP) followed by cataract surgery.

Methods: Thirteen APAC eyes (13 patients) were enrolled in this prospective case series as study group. All patients underwent emergency TCP (20 pulses of $2000 \mathrm{~mW}$ during $2000 \mathrm{~ms}$ applied to the inferior quadrant) followed by scheduled cataract surgery. They were compared to 13 age- and gender-matched patients treated with emergency phacotrabeculectomy. We recorded intraocular pressure (IOP), best corrected visual acuity (BCVA), and complications, and several ultrasound biomicroscopy (UBM) parameters before and after TCP.

Results: In the study group, IOP decreased from $51.5 \pm 7.0 \mathrm{mmHg}$ (mean \pm standard deviation) before TCP to $16.4 \pm$ $5.4 \mathrm{mmHg} 1$ day after TCP $(P<0.001)$. At 6 months, there was no significant difference in IOP between the study group $(14.0 \pm 3.4 \mathrm{mmHg})$ and control group $(16.7 \pm 4.3 \mathrm{mmHg} ; P=0.090)$; IOP lowering medications were used by $0 / 13$ in the study group and $2 / 13$ patients in the control group $(P=0.48)$. At 6 months, there was no significant difference in BCVA between the study group and the control group (20/25 (20/200 to 20/25) and 20/30 (20/50 to 20/25), respectively; $P=1.0)$. The UBM parameters anterior chamber depth $(P=0.016)$, angle-opening distance at $500 \mu \mathrm{m}(P=0.011)$, and maximum ciliary body thickness $(P<0.001)$ increased significantly while the iris-ciliary process distance decreased significantly $(P=0.020)$ after TCP.
\end{abstract}

Conclusions: TCP effectively lowers IOP and modifies the anterior chamber morphology in APAC; TCP followed by cataract surgery can be considered an alternative to treat refractory APAC but needs further evaluation.

Trial registration: This project was registered in Chinese Clinical Trial Registry (ChiCTR1800017475) at July, 31, 2018 (http://www.chictr.org.cn/edit.aspx?pid=29629\&htm=4).

Keywords: Acute primary angle closure, Transscleral cyclophotocoagulation, Ultrasound biomicroscopy

\footnotetext{
* Correspondence: tjykzx@aliyun.com

${ }^{+}$Wei Liu and Luning Qin contributed equally to this work.

${ }^{1}$ Tianjin Key Laboratory of Retinal Functions and Diseases, Tianjin

International Joint Research and Development Centre of Ophthalmology and

Vision Science, Eye Institute and School of Optometry, Tianjin Medical

University Eye Hospital, 251 Fukang Road, Nankai District, Tianjin 300384,

China

Full list of author information is available at the end of the article
}

(c) The Author(s). 2020 Open Access This article is licensed under a Creative Commons Attribution 4.0 International License, which permits use, sharing, adaptation, distribution and reproduction in any medium or format, as long as you give appropriate credit to the original author(s) and the source, provide a link to the Creative Commons licence, and indicate if changes were made. The images or other third party material in this article are included in the article's Creative Commons licence, unless indicated otherwise in a credit line to the material. If material is not included in the article's Creative Commons licence and your intended use is not permitted by statutory regulation or exceeds the permitted use, you will need to obtain permission directly from the copyright holder. To view a copy of this licence, visit http://creativecommons.org/licenses/by/4.0/. The Creative Commons Public Domain Dedication waiver (http://creativecommons.org/publicdomain/zero/1.0/) applies to the data made available in this article, unless otherwise stated in a credit line to the data. 


\section{Background}

Acute primary angle closure (APAC) is a common ophthalmic emergency with the rising rapidly to extremely high levels because of a sudden obstruction of the anterior chamber angle [1]. APAC patients often have typical symptoms, including blurred vision, ocular pain, headache, nausea, and vomiting. Immediate aggressive management is required to prevent irreversible damage to the eye and optic nerve and to relieve the excruciating symptoms of the patient [2]. However, the current treatment options for APAC do not always timely result in a sufficiently lowered IOP.

The current treatment strategy for APAC is to lower IOP by both topical and systemic medications followed by laser peripheral iridoplasty or iridotomy, and finally removal of the lens. However, this strategy has several limitations. The IOP of a significant proportion of APAC eyes cannot be controlled successfully by medical treatment, while laser application is often limited by corneal edema [3]. If the conventional treatment fails to reduce the IOP, surgical procedures in the acute phase have to be considered. However, performing acute surgery on APAC eyes is technically challenging and may increase the risk of complications because of the presence of corneal edema, inflammation, shallow anterior chamber, floppy iris and unstable lens [3].

In our previous study [4], and some other reports [5-7], diode laser transscleral cyclophotocoagulation (TCP) showed its IOP-lowering effects in eyes with APAC. However, the underlying mechanism remains unclear. In this study, we aimed to evaluate the safety and efficacy of TCP followed by cataract surgery on treating APAC. We also compared several ultrasound biomicroscopy (UBM) parameters to explore the underlying mechanisms.

\section{Methods \\ Patients}

This project was registered in Chinese Clinical Trial Registry (ChiCTR1800017475). Consecutive, medically unresponsive APAC patients (study group) from Tianjin Medical University Eye Hospital were prospectively enrolled. All the patients underwent emergency TCP followed by cataract surgery and were followed for 6 months. They underwent a thorough ocular examination, including an IOP measurement, a measurement of the best-corrected visual acuity (BCVA), slit-lamp biomicroscopy, ultrasonography B-scan, and UBM, before and 1 day after TCP. Any complications during and after the TCP were noted. IOP was measured by non-contact tonometry (Full Auto Tonometer TX-F, Canon, Japan) three times and the average value was recorded. This study was approved by the ethics committee of Tianjin
Medical University Eye Hospital (2018KY-03) and followed the tenets of the Declaration of Helsinki. Informed consent was obtained from all subjects after explanation of the nature and possible consequences of the study.

Thirteen age- and gender-matched medically unresponsive APAC patients (control group) who underwent emergency phacotrabeculectomy (the historical default approach in our hospital) without TCP were retrospectively collected. The clinical outcome was compared between the two groups.

\section{Transscleral cyclophotocoagulation}

All TCPs were performed by the same experienced surgeon (WL). TCP was performed using the OcuLight SLx $810 \mathrm{~nm}$ diode laser photocoagulator with the handheld fiberoptic G-probe (Iris Medical Instruments, Mountain View, CA, USA) under local anesthesia $(2 \mathrm{ml}$ of $2 \%$ lidocaine as a retrobulbar injection). The same laser protocol was used for all the patients: power 2000 $\mathrm{mW}$, duration $2000 \mathrm{~ms}$, number of applications 20. Laser applications were spaced evenly over the inferior quadrant (90 degrees), which implies overlapping rather than strictly adjacent shots. This deviates from the default approach in non-acute, open-angle situations, where the laser spots are given over 360 degrees - an approach that did not lower the IOP sufficiently in medically unresponsive APAC eyes in an earlier study [4]. We hypothesized that shrinkage of the ciliary body could be the mechanism and if so, a high dose applied to only a part of the ciliary body should be sufficient whilst having the advantage that the total dose would not increase, thus avoiding excessive inflammation. We chose the inferior quadrant for this, as that quadrant is the deepest in a normal eye, being the logical place to treat in case of closure. After TCP, patients got tobramycin and dexamethasone eye drops (TobraDex, S.A. Alcon-Couvreur N.V., Rijksweg, Puurs, Belgium) 4 times per day during the interval between $\mathrm{TCP}$ and the following cataract surgery.

\section{Ultrasound biomicroscopy and analysis}

The anterior chamber configuration was determined before and 1 day after TCP using UBM (Suoer SW-3200 L; Tianjin, China) and a $50-\mathrm{MHz}$ transducer probe by one experienced operator (LQ). Examinations were performed under the same room illumination and using the same fixation target on the ceiling for the fellow eye, with the patient in the supine position. After topical anesthesia, a plastic eyecup filled with physiologic saline was mounted on the globe without compressing the globe. Images were obtained at the center of the pupil, at the superior and inferior angles of the anterior chamber in the vertical meridian, and at the temporal and 
nasal angles of the anterior chamber in the horizontal meridian.

All captured UBM images were analyzed using the software by a single observer (WL), who was masked to all clinical data, including whether a particular measurement was performed pre- or post-TCP. The UBM parameters measured in this study included the traditional parameters defined by Pavlin et al. $[8,9]$ and newly developed parameters described in recent studies [10-15]. From the images centered on the pupil, two parameters were determined: (1) anterior chamber depth (ACD; the axial distance from the corneal endothelium to the anterior lens surface) and (2) pupil diameter (PD; the shortest distance between the pupil edges of the iris cross-sections). From the radial images at the superior, inferior, nasal, and temporal positions, six parameters were measured (Fig. 1): (1) angle-opening distance at $500 \mu \mathrm{m}$ (AOD500; the distance from the corneal endothelium to the anterior iris perpendicular to a line drawn along the trabecular meshwork $500 \mu \mathrm{m}$ from the sclera spur), (2) iris thickness at $500 \mu \mathrm{m}$ (IT500; iris thickness at $500 \mu \mathrm{m}$ from the sclera spur [point b]), (3) trabecularciliary process distance (TCPD; the length of a line extending from the corneal endothelium $500 \mu \mathrm{m}$ anterior to the sclera spur [point a] toward the ciliary process [point d]), (4) iris-ciliary process distance (ICPD; from the posterior surface of the iris $500 \mu \mathrm{m}$ anterior to the sclera spur [point c] toward the ciliary process [point d]), (5) maximum ciliary body thickness (CBTmax; the distance from the innermost point of the ciliary body to the inner wall of the sclera or its extended line), and (6) the iris curvature (IC; the perpendicular distance from a line between the most central to the most peripheral points of the iris pigment epithelium to the posterior iris surface at the point of greatest convexity).

\section{Cataract surgery}

Cataract surgery was scheduled after TCP to definitely widen the narrow angle. Cataract extraction was performed after the resolution of cornea edema and anterior chamber inflammation. All the surgeries were performed by the same experienced surgeon (JJ). After topical anesthesia with 0.4\% Oxybuprocaine Hydrochloride (Santen, Japan), a small limbal paracentesis was performed at the 2 o'clock and then a superior clear corneal incision was made with $3.0-\mathrm{mm}$ keratome. The chamber was immediately deepened with ocular viscoelastic devices. After the continuous curvilinear capsulorhexis, the nucleus was removed with a standard four-quadrant, "divide and conquer" phacoemulsification technique. The cortical remnants was then removed, and the intraocular lens was placed in the capsular bag. The viscoelastic agent was completely aspirated from the anterior chamber at the end of the surgery.

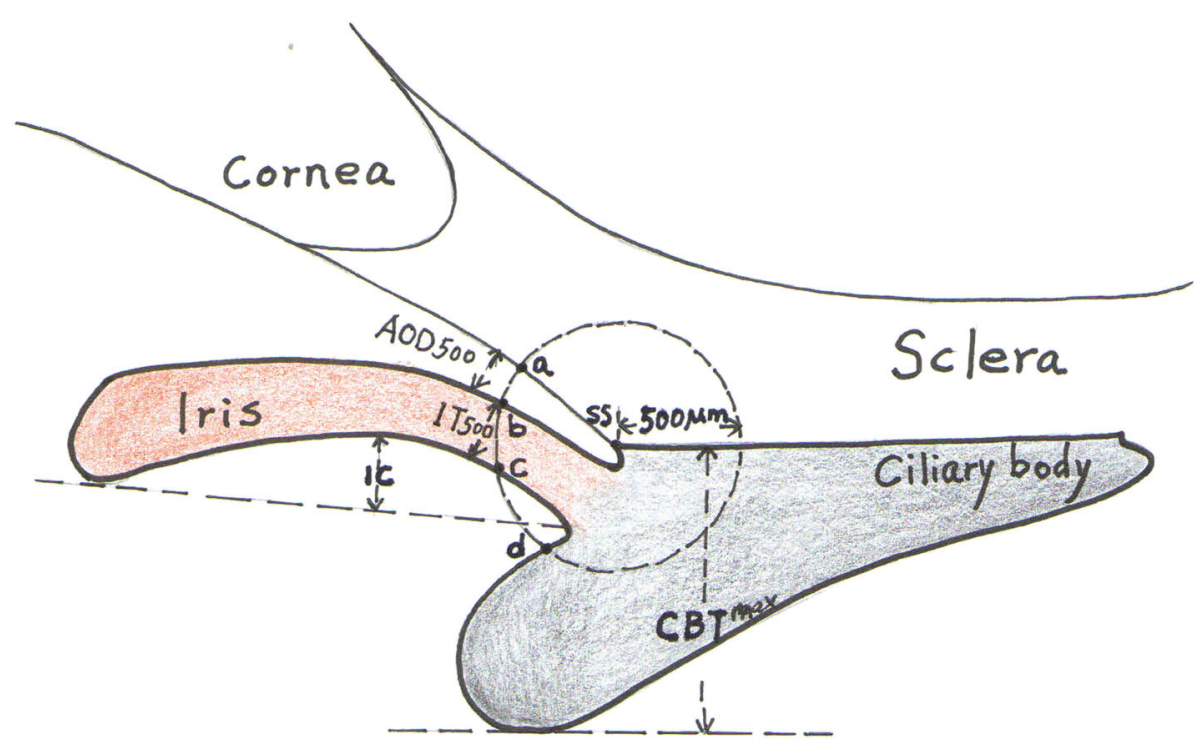

Fig. 1 Schematic diagram of the ultrasound biomicroscopy parameters. A circle with a radius of $500 \mu \mathrm{m}$ centered on the sclera spur (SS) is drawn. Angle-opening distance at $500 \mu \mathrm{m}$ (AOD500) is the distance from corneal endothelium to the anterior iris perpendicular to a line drawn along the trabecular meshwork $500 \mu \mathrm{m}$ from the SS. Iris thickness at $500 \mu \mathrm{m}$ (IT500) is iris thickness at $500 \mu \mathrm{m}$ from the SS (point b). Trabecularciliary process distance (TCPD) is a line extending from the corneal endothelium $500 \mu \mathrm{m}$ anterior to the sclera spur (point a) toward the ciliary process (point d). Iris-ciliary process distance (ICPD) is the posterior surface of the iris $500 \mu \mathrm{m}$ anterior to the sclera spur (point c) toward the ciliary process (point d). Maximum ciliary body thickness (CBTmax) is the distance from the most inner point of the ciliary body to the inner wall of sclera or its extended line. Iris curvature $(\mathrm{IC})$ is the perpendicular distance from a line between the most central to the most peripheral points of the iris pigment epithelium to the posterior iris surface at the point of greatest convexity 


\section{Phacotrabeculectomy}

All the phacotrabeculectomies were performed by the same experienced surgeon (JJ). A two-site procedure was used. After peribulbar anesthesia with $2 \mathrm{ml}$ of $2 \%$ lidocaine (Shandong Hualu, China), a fornix-based conjunctival flap and a rectangular scleral flap (3 by $4 \mathrm{~mm}$ in size) were performed. Mitomycin-C (Zhejiang Hanhui, China) $0.04 \%$ was applied for $3 \mathrm{~min}$ under the conjunctival and scleral flaps. Then, cataract surgery was performed as described above followed by trabeculectomy and peripheral iridectomy. The scleral flap was closed with two 10-0 nylon sutures (one of which was a releasable suture) and the conjunctival wound was closed with three $10-0$ nylon sutures.

\section{Statistical analysis}

Continuous variables were expressed as mean \pm standard deviation (SD) after confirming normality of the data distribution (Shapiro-Wilk test, $P>0.05$ ); if not normally distributed, we used median and interquartile range (IQR). The number of anti-glaucoma medications and the number of patients with BCVA better than 20/40 after surgery were compared between the two groups using Fisher exact test. Age, axial length, and IOP before and after surgery were compared using independent samples $t$ test between the two groups. Pre- and postTCP observations were compared using a paired samples $t$ test or a Wilcoxon signed-rank test, for parametric and nonparametric data, respectively. A $P$ value of 0.05 or less was considered statistically significant.

\section{Results}

Thirteen APAC eyes of 13 consecutive patients refractory to medical treatment (including 1\% pilocarpine, 2\% carteolol, $0.2 \%$ brimonidine, $1 \%$ brinzolamide, oral methazolamide and intravenous $20 \%$ mannitol, if there were no contraindications) were prospectively enrolled in this study. All patients presented with typical APAC symptoms and signs, including blurred vision, severe ocular pain, headache, nausea, vomiting, corneal edema, unresponsive dilated pupil, shallow anterior chamber, and elevated IOP. All these patients underwent emergency TCP followed by scheduled cataract surgery (interval time: 5-10 days, median: 7 days). Table 1 presents the characteristics of the study group and control group.

In the study group, the IOP decreased significantly from $51.5 \pm 7.0 \mathrm{mmHg}$ (mean \pm standard deviation) before TCP to $16.4 \pm 5.4 \mathrm{mmHg}$ at day 1 after TCP $(P<$ 0.001). At day 1 after the TCP, the excruciating symptoms had disappeared and the corneal edema had been resolved in all patients (Fig. 2). The IOP of the study group before cataract surgery $(17.2 \pm 4.8$ $\mathrm{mmHg}$ ) was significantly lower than that of the control group before emergency phacotrabeculectomy $(40.1 \pm 5.7 \mathrm{mmHg} ; \mathrm{P}<0.001)$. At 6 months after surgery, the IOP was $14.0 \pm 3.4 \mathrm{mmHg}$ and $16.7 \pm 4.3$ $\mathrm{mmHg}$ in the study group and control group, respectively $(P=0.090)$. At 6 months after surgery, there were no patients in the study group and 2 patients in the control group who needed anti-glaucoma medications to control the IOP $(P=0.48)$.

In the study group, the BCVA of the patients ranged from hand movement to 20/50 (with a median value of finger counting) before TCP and improved in all patients at day 1 after TCP (ranging from finger counting to 20/ 30; median value 20/60). The single patient with a BCVA of finger counting after TCP was evidenced to have severe glaucomatous neuropathy when the cornea was clear enough to check the fundus. At 6 months after surgery, BCVA ranged from 20/200 to 20/25 (median value $20 / 25$ ) in the study group and from $20 / 50$ to $20 / 25$ (median value 20/30) in the control group. There were 10 patients in the study group and 9 patients in the control group whose BCVA was better than 20/40 at 6 months after surgery $(P=1.0)$.

Table 2 shows the effect of TCP on the 8 UBM parameters studied. A significant increase was found for ACD, AOD500, and CBTmax; a significant decrease was found for ICPD. Figure 3 shows the effect per quadrant for the 6 parameters that were studied for each quadrant separately. As can be seen in this figure, the global changes (as depicted in Table 2) in especially AOD500 and to a lesser extent also in ICPD were mainly due to changes in the, treated, inferior quadrant (Fig. 4) whereas the global change in CBTmax was due to changes in all quadrants.

There were no complications during the TCP procedure. After the TCP, anterior chamber cells were present

Table 1 Baseline demographics of the study participants

\begin{tabular}{llll}
\hline & Study group & Control group & $P$ value \\
\hline Age (years) & $67.6 \pm 4.7(58-75)$ & $69.3 \pm 3.9(61-74)$ & 0.16 \\
Gender, female & $9(69 \%)$ & $10(77 \%)$ & 0.50 \\
Axial length $(\mathrm{mm})$ & $22.13 \pm 0.61(20.90-22.90)$ & $22.25 \pm 0.48(21.13-22.87)$ & 0.29 \\
Eye, right & $7(54 \%)$ & $6(46 \%)$ & 0.50 \\
\hline
\end{tabular}

Presented as mean \pm standard deviation (range) or number (percentage) 


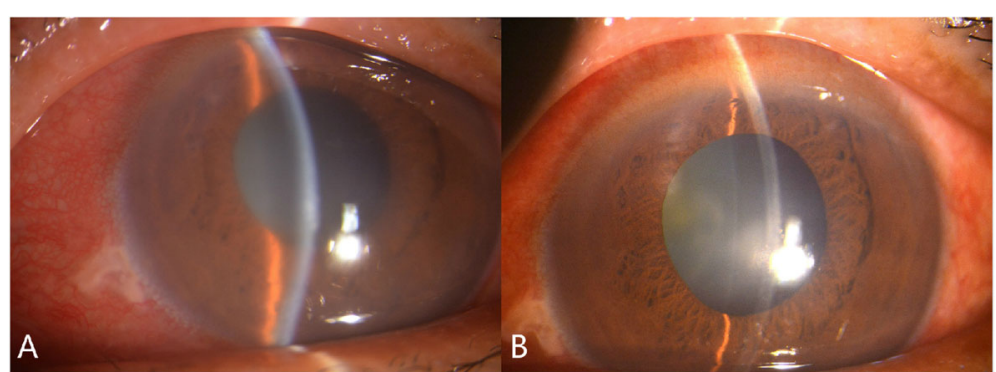

Fig. 2 Anterior segment photograph before and after transscleral cyclophotocoagulation (TCP). A: severe conjunctival congestion and cornea edema before TCP. B: mild conjunctival congestion and clear cornea after TCP

in all the patients, which could be part of the APAC attack itself or it could be due to the TCP. A self-limiting ciliochoroidal detachment was found in 5 patients (39\%) by UBM, mostly in the superior and temporal quadrant (Fig. 4). There were no complications during the subsequent cataract surgery or post-cataract surgery. For the control group, there was 1 patient having a posterior lens capsule rupture during phacotrabeculectomy. After surgery, there were 5 patients with a fibrinous membrane in the anterior chamber in the control group, which needed YAG laser to resolve.

\section{Discussion}

In this study, we explored the safety and efficacy of emergency TCP followed by scheduled cataract surgery on treating refractory APAC and described the anterior segment morphological changes of APAC eyes after TCP by UBM, aiming to explore the IOPlowering mechanism of TCP. The acute attack resolved in all included patients with a major lowering of the IOP. The observed anatomical changes were an increase in ACD, inferior AOD500, and CBTmax, and a decrease in ICPD. Outcomes after 6

Table 2 Comparison of UBM parameters before and after TCP

\begin{tabular}{llll}
\hline & Pre-TCP & Post-TCP & $P$ value \\
\hline ACD $(\mathrm{mm})$ & $1.62 \pm 0.29$ & $1.72 \pm 0.30$ & 0.016 \\
PD $(\mathrm{mm})$ & $3.86 \pm 1.32$ & $4.10 \pm 1.19$ & 0.202 \\
AOD500 $(\mathrm{mm})$ & $0.00(0.00-0.00)$ & $0.04(0.01-0.08)$ & 0.011 \\
IT500 $(\mathrm{mm})$ & $0.29 \pm 0.05$ & $0.32 \pm 0.04$ & 0.095 \\
CBTmax $(\mathrm{mm})$ & $0.98 \pm 0.12$ & $1.12 \pm 0.15$ & 0.000 \\
IC (mm) & $0.20 \pm 0.15$ & $0.14 \pm 0.09$ & 0.062 \\
TCPD (mm) & $0.46 \pm 0.10$ & $0.46 \pm 0.05$ & 0.960 \\
ICPD (mm) & $0.21 \pm 0.10$ & $0.15 \pm 0.08$ & 0.020 \\
\hline
\end{tabular}

Presented as mean \pm standard deviation or median (interquartile range); $A C D$ : anterior chamber depth; PD: pupil diameter; AOD500: angle-opening distance at $500 \mu \mathrm{m}$; IT500: iris thickness at $500 \mu \mathrm{m}$; CBTmax: maximum ciliary body thickness; IC: iris curvature; TCPD: trabecular-ciliary process distance; ICPD: irisciliary process distance months regarding $\mathrm{BCVA}$ and IOP control were similar for emergency TCP followed by cataract surgery versus emergency phacotrabeculectomy.

After two reports on TCP in chronic angle closure glaucoma eyes [16, 17], the use of TCP on acute angle closure (AAC) eyes was first reported in 5 AAC cases from two ophthalmic units in the United Kingdom, in which conventional management, including topical and systemic medical treatment, laser iridotomy, and laser iridoplasty, had been unsuccesful [6]. All of their 5 cases achieved a successful control of the IOP and resolution of the acute attack after TCP. TCP was also shown to be safe and effective in reducing IOP in AAC patients secondary to intumescent cataract $[5,7]$. The only published paper of TCP on APAC eyes was from our previous study [4], in which the IOP of medically unresponsive APAC eyes decreased but remained over $21 \mathrm{mmHg}$ after TCP in all eyes. In the present study, however, the IOP of all the patients was well controlled. This difference might be the result of different laser settings and/or treatment location $(20 \times 2000 \mathrm{~ms} \times 2000 \mathrm{~mW}$ in the inferior quadrant in the current study versus $20-40 \times 1000$ $\mathrm{ms} \times 1700-2200 \mathrm{~mW}$ in the inferior 180 degrees in our previous study).

In this study, we tried to uncover the mechanism underlying the observed decrease in IOP after TCP in APAC. One possible mechanism explaining the decrease in IOP is shrinkage of the ciliary body. Histologically, TCP can cause coagulation necrosis, tissue contraction, and focal atrophy of ciliary body [18, 19], and clinically shrinkage can be observed during endocyclophotocoagulation treatment. Shrinkage will lead to a backward movement of the lens-iris diaphragm, deepening of the anterior chamber, and re-opening of an appositionally closed angle. Another possible mechanism is a sudden decrease in aqueous humor production, which may also contribute to the resolution of the pupillary block [1]. We found significant changes in four parameters: ACD (increase), AOD500 

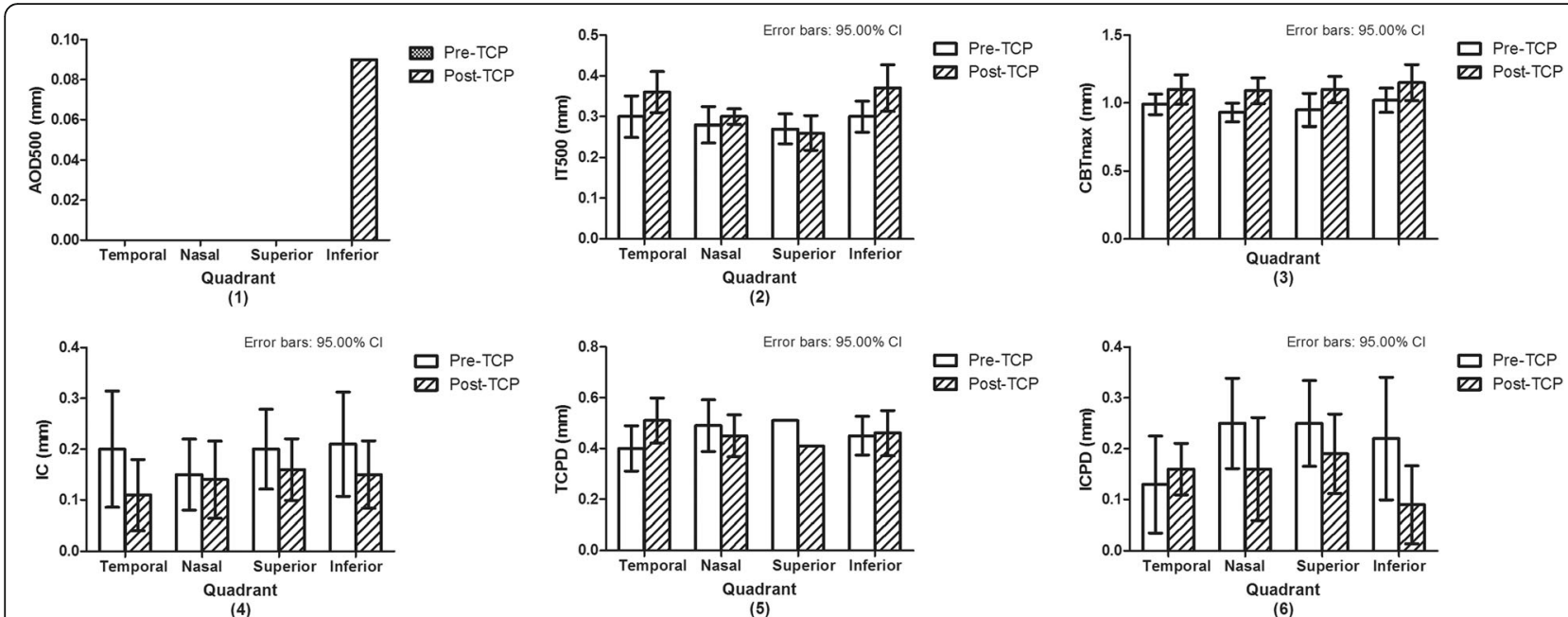

Quadrant
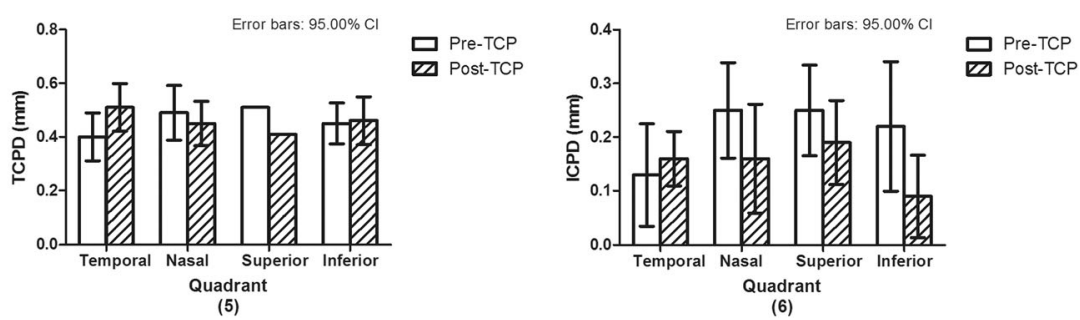

Fig. 3 Comparison of UBM parameters in quadrants before and after transscleral cyclophotocoagulation (TCP). Error bars: 95\% confidential interval. Error bars were not given for the nonparametric data (AOD500). See Fig. 1 for definitions of the parameters (AOD500, IT500, CBTmax, IC, $\mathrm{TCPD}$, and ICPD)

(increase), ICPD (decrease), and CBTmax (increase). The first three are 'in between structures' measures; CBTmax is a 'structure' measure. Both shrinkage and a sudden decrease in aqueous humor production will change the 'in between structures' measures (ACD, AOD500, and ICPD) in the observed direction. These changes describe the disappearance of the block and support the clinical observation: a huge decrease in IOP and clearance of the cornea. At first sight, the observed increase in CBTmax seems to falsify the shrinkage hypothesis, thus favoring a sudden decrease in aqueous humor production as the primary mechanism. However, we only treated inferiorly and the effect of this treatment was at least as effective as the treatment we performed over a larger area in our earlier study [4], arguing against a sudden decrease in aqueous humor production as the primary mechanism. One possibility is an initial shrinkage resolving the pupillary block followed by swelling due to edema formation. UBM observations performed more frequently than a single observation one day after the treatment, as we did, should be able to further our understanding of the exact mechanism of the beneficial effect of TCP in APAC.

The BCVA of all the APAC eyes improved and there was no vision loss after TCP. The main reason of the improvement might be the disappearance of corneal edema after TCP. TCP was initially introduced for IOP reduction in eyes with little or no visual potential. Nowadays, however, TCP is being used widely in eyes with good vision, although the results vary $[16,17,20,21]$. Four studies presenting data on visual acuity changes due to TCP [22-25] reported amounts of vision loss after TCP of 2 lines or more in 24\% [22], 2 lines or more in 31\% [23], unspecified loss in 13\% [24], and 3 lines or more in 14\% [25]. These values are in line with values reported after trabeculectomy or tube surgery [26]. The absence of vision loss in our series suggests that there was no excessive transient IOP rise directly after TCP (we did not measure IOP directly after the procedure; the first measurement was at day 1 after TCP).

Although there were 2 patients in the control group who needed medications to control IOP, there was no significant difference in IOP and BCVA at 6 months after surgery between the two groups. However, the complications of the control group were more severe than study group. This might be resulted from the cloudy cornea and high IOP during phacotrabeculectomy. The poor visibility would increase the risk of intra-operative complications and the dramatic sudden decrease of IOP during surgery would facilitate the occurrence of post-operative fibrinous membrane. Moreover, even without a difference in final BCVA and IOP, the TCP approach has a major logistic advantage as no emergency surgery has to be performed.

We acknowledge the limitations of our study. First, this was not a randomized clinical trial and the sample size was relatively small. Second, IOP was measured by noncontact tonometry. Although a good inter-device agreement between Goldmann tonometry and noncontact tonometry has been reported, [27, 28] Goldmann tonometry is still the gold standard in IOP assessment. However, Goldmann readings also have their limitations with cloudy, edematous corneas, and 


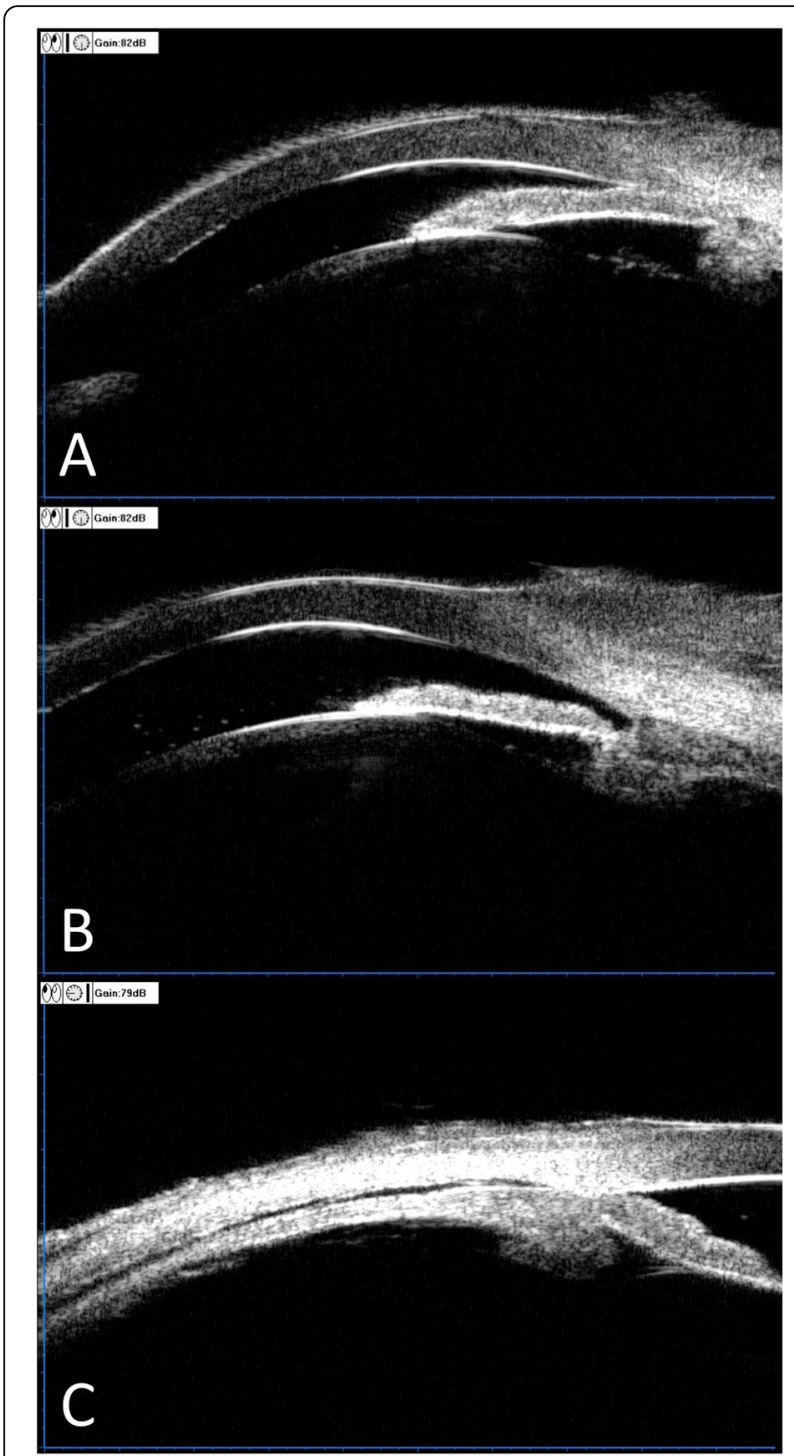

Fig. 4 Ultrasound biomicroscopy image before and after transscleral cyclophotocoagulation (TCP). A: Shallow anterior chamber and closed inferior angle before TCP. B: Deepened anterior chamber and reopened inferior angle after TCP. C: Ciliochoroidal detachment after TCP

given that the treatment-induced IOP changes were very large, the type of tonometer is also less critical. Third, the duration of the IOP-lowering effect of TCP was not evaluated, because TCP was considered a temporary measure to allow for further, final cataract surgery. However, to the best of our knowledge, this is the first study to evaluate the safety and efficacy of TCP followed by cataract surgery on treating APAC and our UBM results can help to better understand the IOP-lowering mechanism of TCP on APAC eyes.

\section{Conclusions}

Our findings suggest that TCP can effectively lower the IOP, deepen the ACD, and widen the inferior angle in
APAC. The presumed mechanism is an initial shrinkage of the involved tissues; UBM more frequently performed early after treatment is needed to further our understanding regarding the exact mechanism. However, even without the mechanism being fully uncovered, TCP could be considered a viable option for the initial treatment of APAC and can facilitate the following cataract surgery. Obviously, a further randomized two-armed clinical trial (TCP followed by cataract extraction versus cataract extraction alone) with larger sample size is needed to confirm the promising results in the present study.

\section{Abbreviations \\ APAC: Acute primary angle closure; IOP: Intraocular pressure; TCP: Transscleral cyclophotocoagulation; UBM: Ultrasound biomicroscopy; BCVA: Best- corrected visual acuity; ACD: Anterior chamber depth; PD: Pupil diameter; AOD500: Angle-opening distance at $500 \mu \mathrm{m}$; IT500: Iris thickness at $500 \mu \mathrm{m}$; TCPD: Trabecular-ciliary process distance; ICPD: Iris-ciliary process distance; CBTmax: Maximum ciliary body thickness; IC: Iris curvature; SD: Standard deviation; IQR: Interquartile range; AAC: Acute angle closure}

\section{Acknowledgements}

Not applicable.

\section{Authors' contributions}

JJ designed and supervised the study; WL drafted the manuscript; WL and LQ collected the data; CX, DH and RG analyzed the data and helped to draft the manuscript; NM.J analyzed the data, reviewed and made meaningful discussion to the manuscript. All authors read and approved the final manuscript.

\section{Funding}

This work was supported by a grant from The Science \& Technology Development Fund of Tianjin Education Commission for Higher Education (grant number: 2016YD09). The funding sponsors were not involved in the analysis design, genotype imputation, data analysis, interpretation of the analysis results, or the preparation, review, or approval of this manuscript.

\section{Availability of data and materials}

The datasets used and/or analysed during the current study are available from the corresponding author on reasonable request.

Ethics approval and consent to participate

This research followed the tenets of the Declaration of Helsinki, informed consent was obtained from the subjects after explanation of the nature and possible consequences of the study. This study was approved by the ethics committee of Tianjin Medical University Eye Hospital.

\section{Consent for publication}

Not applicable.

\section{Competing interests}

The authors declare that they have no competing interests.

\section{Author details}

${ }^{1}$ Tianjin Key Laboratory of Retinal Functions and Diseases, Tianjin International Joint Research and Development Centre of Ophthalmology and Vision Science, Eye Institute and School of Optometry, Tianjin Medical University Eye Hospital, 251 Fukang Road, Nankai District, Tianjin 300384, China. ${ }^{2}$ Department of Ophthalmology, University of Groningen, University Medical Center Groningen, Groningen, The Netherlands. 
Received: 14 April 2020 Accepted: 25 May 2020

Published online: 29 May 2020

\section{References}

1. Zhang X, Liu Y, Wang W, Chen S, Li F, Huang W, et al. Why does acute primary angle closure happen? Potential risk factors for acute primary angle closure. Surv Ophthalmol. 2017;62(5):635-47.

2. Tan AM, Loon SC, Chew PT. Outcomes following acute primary angle closure in an Asian population. Clin Exp Ophthalmol. 2009:37(5):467-72.

3. Chan PP, Pang JC, Tham CC. Acute primary angle closure-treatment strategies, evidences and economical considerations. Eye (Lond). 2019; 33(1):110-9

4. Liu W, Chen $Y, L \vee Y$, Wang $L$, Xing $X$, Liu $A$, et al. Diode laser transscleral cyclophotocoagulation followed by phacotrabeculectomy on medically unresponsive acute primary angle closure eyes: the long-term result. BMC Ophthalmol. 2014;14:26.

5. Manna A, Foster P, Papadopoulos M, Nolan W. Cyclodiode laser in the treatment of acute angle closure. Eye (Lond). 2012;26(5):742-5.

6. Chiam PJ, Sung VCT. The outcome of transscleral cyclophotocoagulation for the management of acute angle closure. Eur J Ophthalmol. 2018; 28(2):188-92.

7. Yusuf $\mathrm{H}$, Shah M, Shaikh A, James CB. Transscleral cyclophotocoagulation in refractory acute and chronic angle closure glaucoma. BMJ Case Rep. 2015; 2015. pii: bcr2015209552

8. Pavlin CJ, Harasiewicz K, Sherar MD, Foster FS. Clinical use of ultrasound biomicroscopy. Ophthalmology. 1991:98(3):287-95.

9. Pavlin CJ, Foster FS. Ultrasound biomicroscopy in glaucoma. Acta Ophthalmol Suppl. 1992;204:7-9.

10. Nongpiur ME, He M, Amerasinghe N, Friedman DS, Tay WT, Baskaran M, et al. Lens vault, thickness, and position in Chinese subjects with angle closure. Ophthalmology. 2011;118(3):474-9.

11. Nongpiur ME, Sakata LM, Friedman DS, He M, Chan YH, Lavanya R, et al. Novel association of smaller anterior chamber width with angle closure in Singaporeans. Ophthalmology. 2010;117(10):1967-73.

12. Wang Z, Huang J, Lin J, Liang X, Cai X, Ge J. Quantitative measurements of the ciliary body in eyes with malignant glaucoma after trabeculectomy using ultrasound biomicroscopy. Ophthalmology. 2014;121(4):862-9.

13. Lin Z, Mou DP, Liang YB, Li SZ, Zhang R, Fan SJ, et al. Reproducibility of anterior chamber angle measurement using the Tongren ultrasound biomicroscopy analysis system. J Glaucoma. 2014;23(2):61-8.

14. Henzan IM, Tomidokoro A, Uejo C, Sakai H, Sawaguchi S, Iwase A, et al. Ultrasound biomicroscopic configurations of the anterior ocular segment in a population-based study the Kumejima Study. Ophthalmology. 2010; 117(9): 1720-1728, 1728.e1.

15. Li M, Chen Y, Chen X, Zhu W, Chen X, Wang X, et al. Differences between fellow eyes of acute and chronic primary angle closure (glaucoma): an ultrasound biomicroscopy quantitative study. PLoS One. 2018;13:e0193006.

16. Lai JS, Tham CC, Chan JC, Lam DS. Diode laser transscleral cyclophotocoagulation in the treatment of chronic angle-closure glaucoma: a preliminary study. J Glaucoma. 2003:12(4):360-4.

17. Lai JS, Tham CC, Chan JC, Lam DS. Diode laser transscleral cyclophotocoagulation as primary surgical treatment for medically uncontrolled chronic angle closure glaucoma: long-term clinical outcomes. J Glaucoma. 2005;14(2):114-9.

18. Brancato R, Leoni G, Trabucchi G, Cappellini A. Histopathology of continuous wave neodymium: yttrium aluminum garnet and diode laser contact transscleral lesions in rabbit ciliary body. A comparative study. Invest Ophthalmol Vis Sci. 1991;32:1586-92.

19. Feldman RM. el-Harazi SM, LoRusso FJ, McCash C, Lloyd WC 3rd, Warner PA. Histopathologic findings following contact transscleral semiconductor diode laser cyclophotocoagulation in a human eye. J Glaucoma. 1997;6:139-40.

20. Kramp K, Vick HP, Guthoff R. Transscleral diode laser contact cyclophotocoagulation in the treatment of different glaucomas, also as primary surgery. Graefes Arch Clin Exp Ophthalmol. 2002;240(9):698-703

21. Grueb M, Rohrbach JM, Bartz-Schmidt KU, Schlote T. Transscleral diode laser cyclophotocoagulation as primary and secondary surgical treatment in primary open-angle and pseudoexfoliatve glaucoma. Long-term clinical outcomes. Graefes Arch Clin Exp Ophthalmol. 2006;244(10):1293-9.

22. Rotchford AP, Jayasawal R, Madhusudhan S, Ho S, King AJ, Vernon SA. Transscleral diode laser cycloablation in patients with good vision. $\mathrm{Br} J$ Ophthalmol. 2010;94(9):1180-3.
23. Ghosh S, Manvikar S, Ray-Chaudhuri N, Birch M. Efficacy of transscleral diode laser cyclophotocoagulation in patients with good visual acuity. Eur J Ophthalmol. 2014;24(3):375-81.

24. Ansari E, Gandhewar J. Long-term efficacy and visual acuity following transscleral diode laser photocoagulation in cases of refractory and nonrefractory glaucoma. Eye (Lond). 2007;21(7):936-40.

25. Wilensky JT, Kammer J. Long-term visual outcome of transscleral laser cyclotherapy in eyes with ambulatory vision. Ophthalmology. 2004;111(7): 1389-92.

26. Junoy Montolio FG, Müskens RPHM, Jansonius NM. Influence of glaucoma surgery on visual function: a clinical cohort study and meta-analysis. Acta Ophthalmol. 2019;97(2):193-9.

27. Kato Y, Nakakura S, Matsuo N, Yoshitomi K, Handa M, Tabuchi H, et al. Agreement among Goldmann applanation tonometer, iCare, and Icare PRO rebound tonometers; non-contact tonometer; and Tonopen XL in healthy elderly subjects. Int Ophthalmol. 2018;38(2):687-96.

28. Yildiz A, Yasar T. Comparison of Goldmann applanation, non-contact, dynamic contour and tonopen tonometry measurements in healthy and glaucomatous eyes, and effect of central corneal thickness on the measurement results. Med Glas (Zenica). 2018;15(2):152-7.

\section{Publisher's Note}

Springer Nature remains neutral with regard to jurisdictional claims in published maps and institutional affiliations.
Ready to submit your research? Choose BMC and benefit from:

- fast, convenient online submission

- thorough peer review by experienced researchers in your field

- rapid publication on acceptance

- support for research data, including large and complex data types

- gold Open Access which fosters wider collaboration and increased citations

- maximum visibility for your research: over $100 \mathrm{M}$ website views per year

At $\mathrm{BMC}$, research is always in progress.

Learn more biomedcentral.com/submissions 